Pacific

Journal of

Mathematics

\title{
CONDUCTORS OF WILDLY RAMIFIED COVERS, III
}

Rachel J. Pries 


\title{
CONDUCTORS OF WILDLY RAMIFIED COVERS, III
}

\author{
RACHEL J. PRIES
}

Consider a wildly ramified $G$-Galois cover of curves $\phi: Y \rightarrow$ $X$ branched at only one point over an algebraically closed field $k$ of characteristic $p$. In this paper, given $G$ such that the Sylow $p$-subgroups of $G$ have order $p$, I show it is possible to deform $\phi$ to increase the conductor at a wild ramification point. As a result, I prove that all sufficiently large conductors occur for covers $\phi: Y \rightarrow \mathbb{P}_{k}^{1}$ branched at only one point with inertia $\mathbb{Z} / p$. For the proof, I show there exists such a cover with small conductor under an additional hypothesis on $G$ and then use deformation and formal patching to transform this cover.

\section{Introduction.}

1.1. Results. Let $X$ be a smooth connected proper curve with marked points $\left\{x_{i}\right\}$ over an algebraically closed field $k$ of characteristic $p$. Consider a Galois cover $\phi: Y \rightarrow X$ of smooth connected curves branched only at $\left\{x_{i}\right\}$. Abhyankar's Conjecture (proved by Raynaud [10] and Harbater [4]) determines exactly which groups $G$ can be the Galois group of $\phi$. An open problem is to determine which inertia groups and filtrations of higher ramification groups can be realized for such a cover $\phi$. More simply, it is unknown which integers can be realized as the genus of $Y$.

The main results of this paper are for the case that $\phi: Y \rightarrow \mathbb{P}_{k}^{1}$ is branched at only one point. Such covers exist if and only if $G$ is a quasi- $p$ group, which means that $G$ is generated by $p$-groups. Harbater $[3]$ proved that the Sylow $p$-subgroups of $G$ can be realized as the inertia groups of such a cover $\phi$. Under the assumption that the Sylow $p$-subgroups of $G$ have order $p$, the filtration of higher ramification groups is determined by one integer $j$ for which $p \nmid j$, namely by the lower jump or conductor. In Theorem 3.2.4, I prove in this case that all sufficiently large conductors occur for such covers of the affine line. Theorem 3.2.4 involves the concept of the $p$-weight which is defined in Section 3.1.

Theorem 3.2.4. Let $G$ be a finite quasi-p group whose Sylow p-subgroups have order $p$. There exists an integer $J$ depending explicitly on $p$, the $p$ weight of $G$, and the exponent of the normalizer of a Sylow p-subgroup of $G$ with the following property: If $j \geq J$ and $p \nmid j$ then there exists a G-Galois 
cover $\phi: Y \rightarrow \mathbb{P}_{k}^{1}$ branched at only one point over which it has inertia group $\mathbb{Z} / p$ and conductor $j$.

The first part of the proof is to show that all sufficiently large conductors will occur. To do this, I show the following more general result in Theorem 2.2.2: Suppose $\phi: Y \rightarrow X$ is a $G$-Galois cover with inertia group $I$ of the form $\mathbb{Z} / p \rtimes \mu_{m}$ and conductor $j$ at a ramification point; then it is possible to deform $\phi$ to increase this conductor. To do this, I construct a family of covers so that $\phi$ is isomorphic to the normalization of one fibre of the family. The techniques consist of local deformations and formal patching theorems of Harbater and Stevenson [5]. Since $k$ is algebraically closed, it is then possible to use another fibre of this family to find another cover with the same group $G$ and inertia group $I$ but with a larger conductor. For certain applications, it is necessary to use ramification data of two covers and deform semi-stable curves in order to enlarge the Galois group and to change the inertia group, while simultaneously enlarging the conductor; see Theorem 2.3.7.

The second part of the proof is to find a relatively small integer $J$ (depending only on the group theory of $G$ ) for which there exists a $G$-Galois cover $\phi: Y \rightarrow \mathbb{P}_{k}^{1}$ branched at only one point over which it has inertia group $\mathbb{Z} / p$ and conductor $J$. For this I use the following result which says roughly speaking that there exists such a cover of the affine line with very small conductor when $G$ has $p$-weight one. (See 3.2.1 for the definition of $j_{\min }(I)$, which is a small set of integers depending only on $I$ and not on $G$ consisting of the minimal possible conductors for a cover of the affine line with inertia $I \simeq \mathbb{Z} / p \rtimes \mu_{m}$.)

Theorem 3.2.2. Let $G$ be a finite quasi-p group of $p$-weight one whose Sylow $p$-subgroups have order $p \neq 2$. For some $I \simeq \mathbb{Z} / p \rtimes \mu_{m} \subset G$ and some $j \in j_{\min }(I)$, there exists a $G$-Galois cover $\phi: Y \rightarrow \mathbb{P}_{k}^{1}$ of smooth connected curves branched at only one point over which it has inertia group $I$ and conductor $j$. In particular, genus $(Y) \leq 1+|G|(p-1) / 2 p$.

This result was announced in [7]. The idea behind its proof is to reverse the process in Section 2 to decrease the conductor of a $G$-Galois cover $\phi$ : $Y \rightarrow \mathbb{P}_{k}^{1}$ branched at only one point. This is done by analyzing the stable model of a family of covers with bad reduction over an equal characteristic discrete valuation ring. This is motivated by the work of Raynaud [11] in unequal characteristic.

1.2. Notation and background. Let $k$ be an algebraically closed field of characteristic $p$. Let $R \simeq k[[t]]$ be an equal characteristic complete discrete valuation ring with residue field $k$ and fraction field $K \simeq k((t))$. For each $m \in \mathbb{N}$ with $\operatorname{gcd}(m, p)=1$, choose an $m$ th root of unity $\zeta_{m} \in k$ such that 
$\zeta_{m_{2}}=\zeta_{m_{2} m_{1}}^{m_{1}}$. Let $G$ be a finite group and let $S$ be a chosen Sylow $p$ subgroup of $G$. The group $G$ is quasi-p if it is generated by all its Sylow $p$-subgroups.

If $X$ is a scheme over $R$, we assume that the morphism $f: X \rightarrow \operatorname{Spec}(R)$ is separated, flat and of finite type. If $\xi$ is a point of a scheme $X$, the germ $\hat{X}_{\xi}$ of $X$ at $\xi$ is defined to be the spectrum of the complete local ring of functions of $X$ at $\xi$. Suppose a scheme $X$ is reduced and connected, but not necessarily irreducible. A morphism $\phi: Y \rightarrow X$ of schemes is a (possibly branched) cover if $\phi$ is finite and generically separable. A $G$-Galois cover is a cover $\phi: Y \rightarrow X$ along with a choice of homomorphism $G \rightarrow \operatorname{Aut}_{X}(Y)$ by which $G$ acts simply transitively on each generic geometric fibre of $\phi$ (again allowing branching). If $\phi: Y \rightarrow X$ is a $G$-Galois cover and $G \subset G^{\prime}$, define the induced cover $\operatorname{Ind}_{G}^{G^{\prime}}(Y) \rightarrow X$ to be the disconnected $G^{\prime}$-Galois cover consisting of $\left(G^{\prime}: G\right)$ copies of $Y$ indexed by the left cosets of $G$ with the induced action of $G^{\prime}$.

Consider a wildly ramified $G$-Galois cover of curves $\phi: Y \rightarrow X$ with branch locus $B$. See [13, Chapter IV] for information about the higher ramification groups of $\phi$. In particular, suppose $\xi \in B$ is a closed point and $\eta \in \phi^{-1}(\xi)$. The inertia group $I$ of $\phi$ at $\eta$ is of the form $I=P \rtimes \mu_{m}$ where $P$ is a $p$-group and $p \nmid m$. In the case that $I \simeq \mathbb{Z} / p \rtimes \mu_{m}$, the conductor of $\phi$ at $\eta$ is the integer $j=\operatorname{val}\left(q\left(\pi_{\eta}\right)-\pi_{\eta}\right)-1$ where $\pi_{\eta}$ is a uniformizer of $Y$ at $\eta$ and $q$ has order $p$ in $I$. In this case, the conductor $j$ is the unique lower jump in the filtration of higher ramification groups and the upper jump is $\sigma=j / m \in \mathbb{Q}$. Up to isomorphism, these objects do not depend on the choice of $\eta$ above $\xi$. If $\xi$ is not a closed point of $X$, the inertia group, filtration of higher ramification groups, and conductor for $\phi$ at $\eta$ are the corresponding objects over the generic point of $\eta$.

This paper frequently uses the following technique of Harbater and Stevenson [5] (also see [9] for the case that $R \not z k[t]]$ ). Let $X$ be a projective $k$-curve that is connected and reduced but not necessarily irreducible. Let $\mathbb{S}$ be a finite closed subset of $X$ which contains the singular locus of $X$.

Definition 1.2.1. A thickening problem of covers for $(X, \mathbb{S})$ consists of the following data:

(a) A cover $f: Y \rightarrow X$ of geometrically connected reduced projective $k$-curves;

(b) for each $s \in \mathbb{S}$, a Noetherian normal complete local domain $R_{s}$ containing $R$ such that $t$ is contained in the maximal ideal of $R_{s}$, along with a finite generically separable $R_{s}$-algebra $A_{s}$;

(c) for each $s \in \mathbb{S}$, a pair of $k$-algebra isomorphisms $F_{s}: R_{s} /(t) \rightarrow \hat{\mathcal{O}}_{X, s}$ and $E_{s}: A_{s} /(t) \rightarrow \hat{\mathcal{O}}_{Y, s}$ which are compatible with the inclusion morphisms. 
Definition 1.2.2. A thickening problem is $G$-Galois if $f$ and $R_{s} \subset A_{s}$ are $G$-Galois and the isomorphisms $F_{s}$ are compatible with the $G$-Galois action (for all $s \in \mathbb{S}$ ). A thickening of $X$ is a projective normal $R$-curve $X^{*}$ such that $X_{k}^{*} \simeq X$. A thickening problem is relative if the data for the problem also includes a thickening $X^{*}$ of $X$, so that $X^{*}$ is a trivial deformation of $X$ away from $\mathbb{S}$ and so that the pullback of $X^{*}$ over the complete local ring at a point $s \in \mathbb{S}$ is isomorphic to $R_{s}$.

Definition 1.2.3. A solution to a thickening problem of covers is a cover $f^{*}: Y^{*} \rightarrow X^{*}$ of projective normal $R$-curves, where $X^{*}$ is a thickening of $X$, whose closed fibre is isomorphic to $f$, whose pullback to the formal completion of $X^{*}$ along $X^{\prime}=X-\mathbb{S}$ is a trivial deformation of the restriction of $f$ over $X^{\prime}$, and whose pullback over the complete local ring at a point $s \in \mathbb{S}$ is isomorphic to $R_{s} \subset A_{s}$ (and such that everything is compatible with the isomorphisms above). (Note that $X^{*}$ is a thickening of $X$.)

Theorem 1.2.4 (Harbater, Stevenson). Every (G-Galois) thickening problem for covers has a (G-Galois) solution. The solution is unique if the thickening problem is relative.

Proof. [5, Theorem 4].

\section{Deformation of covers.}

Consider a $G$-Galois cover $\phi: Y \rightarrow X$ of smooth connected proper $k$-curves. Let $\xi$ be in the branch locus of $\phi$ and let $\eta \in \phi^{-1}(\xi)$. The goal in this section is to deform the cover $\phi$ with precise control over the ramification behavior near $\xi$. To do this it is first necessary to deform the $I$-Galois cover $\hat{\phi}: \hat{Y}_{\eta} \rightarrow \hat{X}_{\xi}$ of germs of curves near $\xi$ with such control. We assume throughout that $p$ strictly divides the order of the inertia group $I$.

2.1. Covers of complete local rings. Let $I$ be a semi-direct product $\mathbb{Z} / p \rtimes \mu_{m}$ with $p \nmid m$. Let $n^{\prime}$ be the order of the prime-to- $p$ part of the center of $I$. Let $U=\operatorname{Spec}(k[[u]])$ and let $b$ be the closed point of $U$. The next results describe the structure of $I$-Galois covers $\phi: X \rightarrow U$ of germs of curves with lower jump $j$ in the filtration of higher ramification groups above $b$.

Definition 2.1.1. Suppose $\phi: X \rightarrow U$ is an $I$-Galois cover of germs of curves such that $X$ is connected but not necessarily normal. Let $r_{1}$ be the number of connected components of the normalization of $X$ and assume that $p \nmid r_{1}$. Define the inertia group of $\phi$ to be the inertia group $I_{1}$ of a closed point in the normalization. The order of $I$ is $p m=p m_{1} r_{1}$ where $p m_{1}$ is the order of $I_{1}$. The conductor (respectively upper jump) of $\phi$ is defined to be the conductor (respectively upper jump) of a ramification point in the normalization. 
Lemma 2.1.2. Let $I \simeq \mathbb{Z} / p \rtimes \mu_{m}$ and let $I_{1} \subset I$ have index $r_{1}$ with $p \nmid r_{1}$. Write $m=r_{1} m_{1}$. Suppose that $\phi: X \rightarrow U$ is an I-Galois cover of connected germs of curves with inertia $I_{1}$ and conductor $j$.

i) There exists an automorphism $A$ of $U$ such that the equations for $A^{*} \phi$ away from $b$ are:

$$
u_{1}^{m}=u^{r_{1}}, x^{p}-x=u_{1}^{-j} .
$$

ii) The Galois action on the generic fibre is given by the following equations for some $\gamma$ with $\operatorname{gcd}(\gamma, m)=1$; (after possibly changing the choice of $q)$ :

$$
c\left(u_{1}\right)=\zeta_{m}^{\gamma} u_{1}, c(x)=\zeta_{m}^{-\gamma j} x, q\left(u_{1}\right)=u_{1}, q(x)=x+1 .
$$

iii) The conductor $j$ satisfies $p \nmid j$ and $\operatorname{gcd}(j, m)=n^{\prime}$. The upper jump is $\sigma=j / m_{1}$.

Proof. First consider the case that $Y$ is normal (i.e., $r_{1}=1$ ). By [8, Lemma 1.4.2], there exists $f(u) \in k[[u]]^{*}$ with degree $j$ so that the equations for $\phi$ away from $u=0$ are $u_{1}^{m}=u$ and $x^{p}-x=u_{1}^{-j} f(u)$. The proof uses Kummer theory and Artin-Schreier Theory. To finish the proof of i) consider the automorphism $A$ of $k[[u]]$ such that $A(u)=u f(u)^{m / j}$. This automorphism exists since $f(u)$ is a $j$ th power in $k[[u]]^{*}$. Then $A\left(u_{1}\right)=u_{1} f(u)^{1 / j}$. After this automorphism of the base, the equations for $A^{*} \phi$ are $u_{1}^{m}=u$ and $x^{p}-x=u_{1}^{-j}$.

Now consider the case that $r_{1} \neq 1$. The normalization of $\phi$ is a disconnected cover whose components are Galois with group $I_{1}$. Thus the normalization has equations:

$$
v^{r_{1}}=1, u_{1}^{m_{1}}=v u, x^{p}-x=u_{1}^{-j} .
$$

The equations for $A^{*} \phi$ in i) are a blow-down of these by the relation $u v=u_{1}^{m_{1}}$. Properties ii)-iii) follow directly from [13, Chapter IV] and $[\mathbf{8}$, Lemma 1.4.2].

The next lemma allows one to induce a given $I_{1}$-Galois cover up to a reducible connected $I$-Galois cover if the restriction from Lemma 2.1.2 (iii) is satisfied. This will be used in Proposition 2.3.4 to patch together covers with different inertia groups.

Lemma 2.1.3. Suppose $I_{1} \subset I \simeq \mathbb{Z} / p \rtimes \mu_{m}$ with index $r_{1}$ where $p \nmid r_{1}$. Let $m=m_{1} r_{1}$. Suppose there exists an $I_{1}$-Galois cover $\phi$ of connected normal germs of curves with conductor $j$. Assume $n^{\prime}=\operatorname{gcd}(m, j)$. Then there exists a connected reducible $I$-Galois cover $\phi^{\text {ind }}$ with conductor $j$ which is isomorphic to $\operatorname{Ind}_{I_{1}}^{I}(\phi)$ away from the closed point. 
Proof. Let $c_{1}=c^{r_{1}}$. By Lemma 2.1.2, there is an automorphism $A$ of $U$ so that the equations for $A^{*} \phi$ are $u_{1}^{m_{1}}=u, x^{p}-x=u_{1}^{-j}$; and its $I_{1^{-}}$ Galois action is given by $c_{1}\left(u_{1}\right)=\zeta_{m_{1}}^{\gamma} u_{1}, c_{1}(x)=\zeta_{m_{1}}^{-\gamma j} x, q\left(u_{1}\right)=u_{1}$ and $q(x)=x+1$ for some $\gamma$ with $\operatorname{gcd}\left(\gamma, m_{1}\right)=1$. The equations for $\operatorname{Ind}_{I_{1}}^{I}\left(A^{*} \phi\right)$ are $v^{r_{1}}=1, u_{1}^{m_{1}}=u v$ and $x^{p}-x=u_{1}^{-j}$.

Let $\phi_{A}^{\text {ind }}$ be the blow down of $\operatorname{Ind}_{I_{1}}^{I}\left(A^{*} \phi\right)$ which identifies the $r_{1}$ ramification points. This yields a connected reducible $I$-Galois cover $\phi_{A}^{\text {ind }}$ whose equations and $I$-Galois action are the same as in Lemma 2.1.2 (i) and (ii). This Galois action is well-defined by the condition on $n^{\prime}$. Let $\phi^{\text {ind }}=$ $\left(A^{-1}\right)^{*} \phi_{A}^{\text {ind }}$ and note that $\phi^{\text {ind }}$ is isomorphic to $\operatorname{Ind}_{I_{1}}^{I}(\phi)$ away from the closed point by construction.

2.2. Deformation of smooth curves. In this section, we show that it is possible to increase the conductor at a branch point while preserving the inertia and Galois group. This result was announced in [7]. Let $R=k[[t]]$ and $K=k((t))$. Let $b$ be the closed point of $U=\operatorname{Spec}(k[[u]])$. Let $U_{R}=$ $\operatorname{Spec}(R[[u]])$ and $U_{K}=U_{R} \times_{R} K=\operatorname{Spec}\left(k[[u, t]]\left[t^{-1}\right]\right)$.

Proposition 2.2.1. Let $I \simeq \mathbb{Z} / p \rtimes \mu_{m}$. Suppose there exists an $I$-Galois cover $\phi: X \rightarrow U$ of normal connected germs of curves with conductor $j$. Then for $i \in \mathbb{N}$ with $p \nmid(j+i m)$, there exists an I-Galois cover $\phi_{R}: X_{R} \rightarrow$ $U_{R}$ of irreducible germs of $R$-curves, whose branch locus consists of only the $R$-point $b_{R}=b \times_{k} R$, such that:

1. The normalization of the special fibre of $\phi_{R}(t=0)$ is isomorphic to $\phi$ away from $b$.

2. The generic fibre $\phi_{K}: X_{K} \rightarrow U_{K}$ of $\phi_{R}$ is an I-Galois cover of normal connected curves whose branch locus consists of only the $K$-point $b_{K}=$ $b_{R} \times_{R} K$ over which it has inertia $I$ and conductor $j+i m$.

Proof. After an automorphism $A$ of $k[[u]]$, the equations for $A^{*} \phi$ are given by: $u_{1}^{m}=u, x^{p}-x=u_{1}^{-j}$. Consider the normal cover $\phi_{R}^{\prime}: X_{R}^{\prime} \rightarrow U_{R}$ given generically by the equations:

$$
u_{1}^{m}=u, x^{p}-x=u_{1}^{-(j+i m)}\left(t+u^{i}\right) .
$$

The $I$-Galois action on the variables is given by the same expressions as on the closed fibre and the cover is irreducible. The curve $X_{R}^{\prime}$ is singular only above the point $(u, t)=(0,0)$. The normalization of the special fibre agrees with $A^{*} \phi$. The cover $\phi_{R}^{\prime}$ is branched only at the $R$-point $u=0$ since $u_{1}=0$ is the only pole of the function $u_{1}^{-(j+i m)}\left(t+u^{i}\right)$. Taking the restriction of $\phi_{K}$ over $\operatorname{Spec}(K[[u]])$ where $t+u^{i}$ is a unit, we see that $\phi_{K}$ has inertia $I$ and conductor $j+i m$ over $b_{K}$. Pulling back the cover $\phi_{R}^{\prime}$ by the automorphism $A^{-1} \times_{k} R$ of $R[[u]]$ changes none of these properties and thus yields the cover $\phi_{R}$. 
Let $X_{k}$ be a proper $k$-curve. The next theorem uses Propositon 2.2.1 and Theorem 1.2.4 to deform a given cover of $X_{k}$ to a family of covers $\phi_{R}$ of $X_{k}$. This family can be defined over a variety $\Theta$ of finite type over $k$. We then specialize to a fibre of the family over another $k$-point of $\Theta$ to get a cover $\phi^{\prime}$ with new ramification data.

Theorem 2.2.2. Suppose there exists a G-Galois cover $\phi: Y \rightarrow X_{k}$ of smooth connected curves with branch locus $B$. Suppose $\phi$ has inertia group $I \simeq \mathbb{Z} / p \rtimes \mu_{m}$ and conductor $j$ above $\xi_{1} \in B$ with $p \nmid m$. Let $i \in \mathbb{N}^{+}$be such that $p \nmid(j+i m)$. Then there exist $G$-Galois covers $\phi_{R}: Y_{R} \rightarrow X_{R}$ and $\phi^{\prime}: Y^{\prime} \rightarrow X_{k}$ such that:

1. The curves $Y_{R}$ and $Y^{\prime}$ are irreducible and $Y_{K}$ and $Y^{\prime}$ are smooth and connected.

2. After normalization, the special fibre $\phi_{k}$ of $\phi_{R}$ is isomorphic to $\phi$ away from $\xi_{1}$.

3. The branch locus of the cover $\phi_{R}$ (respectively $\left.\phi^{\prime}\right)$ consists exactly of the $R$-points $\xi_{R}=\xi \times_{k} R$ (respectively the $k$-points $\xi$ ) for $\xi \in B$.

4. For $\xi \in B, \xi \neq \xi_{1}$, the ramification behavior for $\phi_{R}$ (respectively $\phi^{\prime}$ ) at $\xi_{R}$ (respectively $\xi$ ) is identical to that of $\phi$ at $\xi$.

5. The cover $\phi_{K}$ (respectively $\left.\phi^{\prime}\right)$ has inertia $I$ and conductor $j+i m$ at the $K$-point $\xi_{1, K}$ (respectively at $\left.\xi_{1}\right)$.

6. The genus of $Y^{\prime}$ and of the fibres of $Y_{R}$ is $g_{Y}^{\prime}=\operatorname{genus}(Y)+i|G|(p-$ 1) $/ 2 p$.

Proof. In the notation of Theorem 1.2.4, let $X^{*}=X_{R}$ and let $\mathbb{S}=\left\{\xi_{1}\right\}$. Let $\eta \in \phi^{-1}\left(\xi_{1}\right)$. Consider the $I$-Galois cover $\hat{\phi}: \hat{Y}_{\eta} \rightarrow \hat{X}_{\xi_{1}}$. Applying Proposition 2.2 .1 to $\hat{\phi}$, there exists a deformation $\hat{\phi}_{R}: \hat{Y}_{R} \rightarrow \hat{X}_{R}$ of $\hat{\phi}$ with the desired properties. In particular, $\hat{\phi}_{K}$ has inertia $I \simeq \mathbb{Z} / p \rtimes \mu_{m}$ and conductor $j+i m$ over $\xi_{1, K}$. Consider the inclusion $R_{s} \rightarrow A_{s}$ of rings corresponding to the disconnected $G$-Galois cover $\operatorname{Ind}_{I}^{G}\left(\hat{\phi}_{R}\right)$.

The covers $\phi_{k}$ and $\operatorname{Ind}_{I}^{G}\left(\hat{\phi}_{R}\right)$ and the isomorphism given by Proposition 2.2.1 (1) constitute a relative $G$-Galois thickening problem as in Definition 1.2.1. The (unique) solution to this thickening problem (Theorem 1.2.4) yields the $G$-Galois cover $\phi_{R}: Y_{R} \rightarrow X_{R}$. Recall from Definition 1.2.3 that the cover $\phi_{R}$ is isomorphic to $\operatorname{Ind}_{I}^{G}\left(\hat{\phi}_{R}\right)$ over $\hat{X}_{R, \xi_{1}}$. Also, $\phi_{R}$ is isomorphic to the trivial deformation $\phi_{t r}: Y_{t r} \rightarrow X_{t r}$ of $\phi$ away from $\xi_{1}$. Thus $Y_{R}$ is irreducible since $Y$ is irreducible and $Y_{K}$ is smooth since $Y_{t r, K}$ and $\hat{Y}_{K}$ are smooth.

The data for the cover $\phi_{R}$ is contained in a subring $\Theta \subset R$ of finite type over $k$, with $\Theta \neq k$ since the family is nonconstant. Since $k$ is algebraically closed, there exist infinitely many $k$-points of $\operatorname{Spec}(\Theta)$. The closure $L$ of the locus of $k$-points $x$ of $\operatorname{Spec}(\Theta)$ over which the fibre $\phi_{x}$ is not a $G$-Galois cover of smooth connected curves is closed, [2, Proposition 9.29]. Furthermore, 
$L \neq \operatorname{Spec}(\Theta)$ since $Y_{K}$ is smooth and irreducible. Let $\phi^{\prime}: Y^{\prime} \rightarrow X_{k}$ be the fibre over a $k$-point not in $L$. Note that $Y^{\prime}$ is smooth and irreducible by definition. The other properties follow immediately from the compatibility of $\phi_{R}$ with $\operatorname{Ind}_{I}^{G}\left(\hat{\phi}_{R}\right)$ over $\hat{X}_{R, \xi_{1}}$ and with $\phi_{t r}: Y_{t r} \rightarrow X_{t r}$ away from $\xi_{1}$.

The genus of $Y^{\prime}$ and of the fibres of $Y_{R}$ increases because of the extra contribution to the Riemann-Hurwitz formula. In particular, there are $|G| / m p$ ramification points above $\xi_{1, K}$, each of which has $i m$ extra nontrivial higher ramification groups. Thus the degree of ramification $\operatorname{Deg}\left(\xi_{1}\right)$ over $\xi_{1, K}$ increases by $|G|(\mathrm{im})(p-1) / m p$.

Theorem 2.2.2 can be used to increase the conductor of a cover of proper curves while preserving the inertia and Galois group. For some applications, however, it is necessary to change the Galois group, the prime-to- $p$ part of the inertia group, or the congruence value of the conductor. To do this, it is necessary to deform covers of semistable curves.

2.3. Deformation of semi-stable curves. In this section, we deform covers of semi-stable curves with control over the ramification information. The motivation for Theorem 2.3.7 is that it allows us to use two wildly ramified covers to produce another whose Galois group, inertia group and conductor at a point are determined from the given ones. In Section 3, we use this theorem to produce a cover with complicated Galois group and relatively small conductor. See $[\mathbf{1}]$ for an application in which the prime-to- $p$ part of the inertia group, and the congruence value of the conductor are changed using this theorem. Since the notation involved in Theorem 2.3.7 is complicated, we will start with a corollary.

Corollary 2.3.1. Let $G$ be a group generated by subgroups $G_{1}$ and $G_{2}$. Let $\phi_{1}: X \rightarrow \mathbb{P}_{k}^{1}$ and $\phi_{2}: Y \rightarrow \mathbb{P}_{k}^{1}$ be Galois covers of smooth connected curves each branched at only one point. Suppose $\phi_{i}$ has group $G_{i}$, inertia $I_{i}$, and conductor $j_{i}$ respectively. Then there exists a G-Galois cover $\phi^{\prime}: Y^{\prime} \rightarrow \mathbb{P}_{k}^{1}$ of smooth connected curves, branched at only one point with inertia $I^{\prime}$ and conductor $j^{\prime}$ as follows:

1. Purely wild: Suppose $I_{1}=I_{2}=\mathbb{Z} / p$. Then $I^{\prime}=\mathbb{Z} / p$ and $j^{\prime}=$ $j_{1}+j_{2}+\epsilon$. Here $\epsilon=0$ if $p \nmid\left(j_{1}+j_{2}\right)$; if $p \mid\left(j_{1}+j_{2}\right)$, then $\epsilon=2$ if $p \neq 2$ and $\epsilon=3$ if $p=2$.

2. Admissible: Suppose $I_{1} \simeq I_{2}$ and $j_{1}=\gamma j_{2}$ for some $\gamma \equiv-1 \bmod m$. Write $\gamma=\nu m-1$. Assume that $p \nmid\left(j_{1}+j_{2}\right)$. Then $I^{\prime}=\mathbb{Z} / p$ and $j^{\prime}=\left(j_{1}+j_{2}\right) / m=\nu j_{2}$.

3. Different inertia: More generally, suppose $I_{1} \subset I_{2}=\mathbb{Z} / p \rtimes \mu_{m}$ with index $r$ for some $r$ with $p \nmid r$. Let $e=j_{1} r+j_{2}$ and let $g=$ $\operatorname{gcd}\left(m, e / \operatorname{gcd}\left(j_{1}, j_{2}\right)\right)$. Assume that $j_{1}=\gamma j_{2}$ for some $\gamma$ such that $\operatorname{gcd}(\gamma, m)=1$. Assume that $p \nmid e$. Then $I^{\prime}$ is the unique subgroup of $I_{2}$ with order $\mathrm{pm} / \mathrm{g}$ and $j^{\prime}=e / \mathrm{g}$. 
The second case is called admissible since the prime-to- $p$ ramification disappears.

Proof. The proof is immediate from Theorem 2.3.7 and Proposition 2.3.5.

Notation 2.3.2. Let $U=\operatorname{Spec}(k[[u]])$ and $V=\operatorname{Spec}(k[[v]])$. For $e \in \mathbb{N}^{+}$, we define $\Omega_{u v}^{e}=k[[u, v, t]] /\left(u v-t^{e}\right)$ and let $S_{u v}^{e}=\operatorname{Spec}\left(\Omega_{u v}^{e}\right)$. Let $\iota_{u}: U \rightarrow$ $S_{u v}^{e}$ and let $\iota_{v}: V \rightarrow S_{u v}^{e}$ be the natural inclusions. Let $b \in S_{u v}^{e}$ be the $k$-point $(u, v, t)=(0,0,0)$. Suppose $I \simeq \mathbb{Z} / p \rtimes \mu_{m}=\mathbb{Z} / p \rtimes\langle c\rangle$. Let $n^{\prime}$ be the order of the prime-to- $p$ part of the center of $I$. For $i=1,2$, suppose $I_{i}=\mathbb{Z} / p \rtimes \mu_{m_{i}} \subset I$ with index $r_{i}$ where $p \nmid r_{i}$. Note that $m=m_{1} r_{1}=m_{2} r_{2}$.

Let $\phi_{1}: X \rightarrow U, \phi_{2}: Y \rightarrow V$ be Galois covers of normal connected germs of curves. Suppose the cover $\phi_{i}$ has inertia (and Galois) group $I_{i} \simeq \mathbb{Z} / p \rtimes \mu_{m_{i}}$ and conductor $j_{i}$ for $i=1,2$. Let $g^{\prime}=\operatorname{gcd}\left(j_{1}, j_{2}\right)$. Let $e=j_{1} r_{1}+j_{2} r_{2}$, and let $g=\operatorname{gcd}\left(m, e / g^{\prime}\right)$. Let $e^{\prime}=e / g^{\prime}, j_{1}^{\prime}=j_{1} / g^{\prime}$ and $j_{2}^{\prime}=j_{2} / g^{\prime}$.

Numerical hypotheses: Suppose that $n^{\prime}=\operatorname{gcd}\left(m, j_{1}\right)=\operatorname{gcd}\left(m, j_{2}\right)$. Suppose $p \nmid e$. Suppose that $j_{1}^{\prime} \equiv \gamma j_{2}^{\prime} \bmod m$ for some $\gamma \operatorname{such}$ that $\operatorname{gcd}(\gamma, m)=1$ and $1 \leq \gamma<m$. Suppose that $\operatorname{gcd}\left(j_{2}^{\prime}, m\right)=1$.

The first condition is necessary to dominate each cover by an $I$-Galois cover. The other three conditions imply that $p \nmid(e / g) ; \operatorname{gcd}\left(j_{1}^{\prime}, m\right)=1$; and $g=\operatorname{gcd}\left(m, \gamma r_{1}+r_{2}\right)$. Also, when $j_{1}=\gamma j_{2}$ and $p \nmid(\gamma+1)$ for some $\gamma$ with $\operatorname{gcd}(\gamma, m)=1$ then the three last conditions are satisfied.

Proposition 2.3.4 constructs an $I$-Galois cover $\phi_{R}: W_{R} \rightarrow S_{u v}^{e^{\prime}}$ with specified ramification from the covers $\phi_{1}$ and $\phi_{2}$. Although $W_{R}$ will be flat over $R$ and normal, its special fibre $W_{k}$ will be singular at the point $w=\phi_{R}^{-1}(b)$.

The following lemma will be used in the proof of Proposition 2.3.4:

Lemma 2.3.3. Suppose $\ell=\ell_{1}+\ell_{2}$ with $\ell_{i} \in \mathbb{N}^{+}$and $a \in k$. Then $d_{0} \in \Omega_{u v}^{\ell}$ where

$$
d_{0}=\frac{\left(u+a t^{\ell_{2}}\right)^{\ell}-u^{\ell}-\left(a t^{\ell_{2}}\right)^{\ell}}{u^{\ell_{2}} t} .
$$

Proof. It is sufficient to show that the binomial coefficient $c_{i}=u^{i} t^{\ell_{2}(\ell-i)} / u^{\ell_{2}} t$ $\in \Omega_{u v}^{\ell}$ for $1 \leq i \leq \ell-1$. Since $t^{\ell}=u v$,

$$
c_{i}=u^{i-\ell_{2}}(u v)^{\ell_{2}} t^{-\ell_{2} i-1}=u^{i} v^{\ell_{2}} t^{-\ell_{2} i-1} .
$$

Since $\Omega_{u v}^{\ell}$ is normal, it is sufficient to check that $c_{i}^{\ell} \in \Omega_{u v}^{\ell}$. Here

$$
c_{i}^{\ell}=u^{\ell i} v^{\ell \ell_{2}}(u v)^{-\ell_{2} i-1}=u^{i\left(\ell-\ell_{2}\right)-1} v^{\ell_{2}(\ell-i)-1} .
$$

Thus $c_{i}^{\ell} \in \Omega_{u v}^{\ell}$ since $\ell-\ell_{2} \geq 1$ and $\ell-i \geq 1$. 
Proposition 2.3.4. Consider the pair $\left(\phi_{1}, \phi_{2}\right)$ from in Notation 2.3 .2 satisfying the numerical hypotheses. There exists an I-Galois cover $\phi_{R}: W_{R} \rightarrow$ $S^{e^{\prime}}$ of (possibly disconnected) germs of $R$-curves and an isomorphism $i$ : $S_{u v}^{e^{\prime}} \rightarrow S^{e^{\prime}}$ such that:

1. The branch locus of $\phi_{R}$ consists of one $R$-point, denoted $b_{R}$, which specializes to $b$.

2. After normalization, the pullbacks of the special fibre of $\phi_{R}$ to $U$ and $V$, namely $\iota_{u}^{*} i^{*} \phi_{k}$ and $\iota_{v}^{*} i^{*} \phi_{k}$, are isomorphic to a disjoint union of copies of respectively $\phi_{1}$ and $\phi_{2}$ away from the branch point $b$.

3. The generic fibre $\phi_{K}: X_{K} \rightarrow S_{K}^{e^{\prime}}=S^{e^{\prime}} \times_{R} K$ of $\phi_{R}$ is an I-Galois cover of (possibly disconnected) germs of curves branched at exactly the $K$-point $b_{K}=b_{R} \times{ }_{R} K$.

4. The cover $\phi_{K}$ has $g$ ramification points above $b_{K}$, each with inertia group $I_{K} \simeq \mathbb{Z} / p \rtimes\left\langle c^{g}\right\rangle=\mathbb{Z} / p \rtimes \mu_{m / g}$ and conductor $e / g=\left(j_{1} r_{1}+\right.$ $\left.j_{2} r_{2}\right) / g$.

5. The curve $W_{R}$ is irreducible if and only if $\operatorname{gcd}\left(r_{1}, r_{2}\right)=1$. The curve $W_{K}$ is irreducible if and only if $g=1$.

Proof. The proof is to construct $\phi_{R}$ and then verify its properties.

The equations for $\phi_{1}^{\text {ind }}$ and $\phi_{2}^{\text {ind }}$ : Applying Lemma 2.1.2, there exist automorphisms $A_{u}$ of $k[[u]]$ and $A_{v}$ of $k[[v]]$ which fix the closed points of $U$ and $V$ and such that the pullbacks $A_{u}^{*} \phi_{1}$ and $A_{v}^{*} \phi_{2}$ are given by the equations in Lemma 2.1.2 (i).

Since $n^{\prime}=\operatorname{gcd}\left(m, j_{1}\right)=\operatorname{gcd}\left(m, j_{2}\right)$, Lemma 2.1.3 implies that there exist connected reducible $I$-Galois covers $\phi_{1}^{\text {ind }}$ and $\phi_{2}^{\text {ind }}$ which are isomorphic respectively to $A_{u}^{*} \phi_{1}$ and $A_{v}^{*} \phi_{2}$ away from the branch point. The equations for these covers are:

$$
\begin{aligned}
& \phi_{1}^{\text {ind }}: u_{1}^{m}=u^{r_{1}}, x^{p}-x=u_{1}^{-j_{1}} \\
& \phi_{2}^{\text {ind }}: v_{1}^{m}=v^{r_{2}}, y^{p}-y=v_{1}^{-j_{2}} .
\end{aligned}
$$

After possibly changing $c$ and $q$ once and for all, the $I$-Galois action of $\phi_{1}^{\text {ind }}$ and $\phi_{2}^{\text {ind }}$ is given (for some $\gamma$ such that $\operatorname{gcd}(\gamma, m)=1$ and some $a \in \mathbb{F}_{p}$ ) by:

$$
\begin{array}{lll}
c\left(u_{1}\right)=\zeta_{m} u_{1}, & c(x)=\zeta_{m}^{-j_{1}} x, & q(x)=x+1, \\
c\left(v_{1}\right)=\zeta_{m}^{\gamma} v_{1}, & c(y)=\zeta_{m}^{-\gamma j_{2}} y, & q(y)=y+a .
\end{array}
$$

Note that the normalization of $\phi_{1}^{\text {ind }}$ (resp. $\phi_{2}^{\text {ind }}$ ) is isomorphic to a disjoint union of copies of $A_{u}^{*} \phi_{1}$ (resp. $A_{v}^{*} \phi_{2}$ ) away from the closed point.

The equations for $\phi_{R}$ : Let $g^{\prime}=\operatorname{gcd}\left(j_{1}, j_{2}\right)$ and $e^{\prime}=j_{1}^{\prime} r_{1}+j_{2}^{\prime} r_{2}$ be as in

Notation 2.3.2. There exists $a_{1} \in k$ such that $a_{1}^{g^{\prime}}=a^{m}$ since $k$ is 
algebraically closed. Consider the cover $\phi_{R}^{\prime}: W_{R} \rightarrow S_{u v}^{e^{\prime}}$ given by:

$$
w_{1}^{m}=u^{j_{1}^{\prime} r_{1}}+a_{1} v^{j_{2}^{\prime} r_{2}}+d_{0} t, z^{p}-z=\left(1+d_{1} t\right) w_{1}^{-g^{\prime}} .
$$

For any choice of the variables $d_{0}, d_{1} \in \Omega_{u v}^{e^{\prime}}$, the cover $\phi_{R}^{\prime}$ reduces to $\phi_{1}^{\text {ind }}$ and $\phi_{2}^{\text {ind }}$ on the components of the special fibre. To see this, note that $\bmod (v, t)$ the equations for $\iota_{u}^{*} i^{*} \phi_{R}^{\prime}$ are $w_{1}^{m}=u^{j_{1}^{\prime} r_{1}}$ and $z^{p}-$ $z=w_{1}^{-g^{\prime}}$. After making the identification $w_{1} \mapsto u_{1}^{j_{1}^{\prime}}$ and $z \mapsto x$, a normalization of these equations is isomorphic to $\phi_{1}^{\text {ind }}$. (Specifically, we take a normalization of $u_{1}^{j_{1}^{\prime} m}=u^{j_{1}^{\prime} r_{1}}$ and $x^{p}-x=u_{1}^{-g^{\prime} j_{1}^{\prime}}=u_{1}^{-j_{1}}$.) Likewise, $\bmod (u, t)$ the equations for $\iota_{v}^{*} i^{*} \phi_{R}^{\prime}$ are $w_{1}^{m}=a_{1} v_{2}^{j_{2}^{\prime} r_{2}}$ and $z^{p}-z=w_{1}^{-g^{\prime}}$. After making the identification $w_{1} \mapsto a_{1}^{1 / m} v_{1}^{j_{2}^{\prime}}$ and $z \mapsto y / a$, this simplifies to $v_{1}^{j_{2}^{\prime} m}=v^{j_{2}^{\prime} r_{2}}$ and $y^{p}-y=a\left(a_{1}^{1 / m} v_{1}^{j_{2}^{\prime}}\right)^{-g^{\prime}}=$ $v_{1}^{-j_{2}}$. A normalization of these equations is isomorphic to $\phi_{2}^{\text {ind }}$. By the numerical hypotheses that $j_{1}^{\prime} \equiv \gamma j_{2}^{\prime} \bmod m$ and $\operatorname{gcd}\left(j_{1}^{\prime}, m\right)=1$, there is a well-defined $I$-Galois action on $\phi_{R}^{\prime}$ which reduces correctly:

$$
c\left(w_{1}\right)=\zeta_{m}^{j_{1}^{\prime}} w_{1}, c(z)=\zeta_{m}^{-j_{1}} z, q(z)=z+1 .
$$

In conclusion, after normalization, the pullbacks of the special fibre of $\phi_{R}^{\prime}$ to $U$ and $V$, namely $\iota_{u}^{*} i^{*} \phi_{k}$ and $\iota_{v}^{*} i^{*} \phi_{k}$, are isomorphic to a disjoint union of copies of respectively $A_{u}^{*} \phi_{1}$ and $A_{v}^{*} \phi_{2}$ away from the branch point $b$.

The cover $\phi_{R}$ will be the composition of $\phi_{R}^{\prime}$ with a change of base. Namely, suppose $A_{u}^{-1}(u)=u d_{u}$ and $A_{v}^{-1}(v)=v d_{v}$ for $d_{u} \in k[[u]]^{*}$ and $d_{v} \in k[[v]]^{*}$. Recall that $\Omega_{u v}^{e^{\prime}}=k[[u, v, t]] /\left(u v-t^{e^{\prime}}\right)$. Let $\Omega^{e^{\prime}}=$ $k[[u, v, t]] /\left(u v d_{u} d_{v}-t^{e^{\prime}}\right)$ and let $S^{e^{\prime}}=\operatorname{Spec}\left(\Omega^{e^{\prime}}\right)$. There exists an isomorphism $A: \Omega^{e^{\prime}} \rightarrow \Omega_{u v}^{e^{\prime}}$ which reduces to $A_{u}$ on $U$ and $A_{v}$ on $V$.

Consider the pullback of the cover $\phi_{R}^{\prime}$ by $A$. In other words, consider the cover $\phi_{R}: W_{R} \rightarrow S^{e^{\prime}}$ corresponding to the composition $\Omega^{e^{\prime}} \stackrel{A}{\rightarrow}$ $\Omega_{u v}^{e^{\prime}} \rightarrow \mathcal{O}_{W^{\prime}}$. Most properties for $\phi_{R}$ in Proposition 2.3.4 are automatic by the construction of $\phi_{R}^{\prime}$. Since $A$ is an isomorphism, to finish the proof it is sufficient to verify Properties 3)-5) for $\phi_{R}^{\prime}$.

The branch locus: Note that $e^{\prime}=j_{1}^{\prime} r_{1}+j_{2}^{\prime} r_{2}$ is the sum of two positive integers. Let

$$
d_{0}=\frac{\left(u+a_{2} t_{2}^{j_{2}^{\prime} r_{2}}\right)^{e^{\prime}}-u^{e^{\prime}}-a_{1} t_{2}^{j_{2}^{\prime} r_{2} e^{\prime}}}{u^{j_{2}^{\prime} r_{2}} t} .
$$

By Lemma 2.3.3, $d_{0} \in \Omega_{u v}^{e^{\prime}}$. Rewrite the first equation for $\phi_{R}^{\prime}$ as:

$$
w_{1}^{m}=u^{-j_{2}^{\prime} r_{2}}\left(u^{e^{\prime}}+a_{1}(u v)^{j_{2}^{\prime} r_{2}}+d_{0} t u^{j_{2}^{\prime} r_{2}}\right) .
$$

Since $u v=t^{e^{\prime}}$, this simplifies to: $w_{1}^{m}=u^{-j_{2}^{\prime} r_{2}}\left(u^{e^{\prime}}+a_{1}\left(t^{j_{2}^{\prime} r_{2}}\right)^{e^{\prime}}+\right.$ $\left.d_{0} t u^{j_{2}^{\prime} r_{2}}\right)$. 
For this choice of $d_{0}$ and for $a_{2}=a_{1}^{1 / e}$, the equations on the generic fibre are:

$$
w_{1}^{m}=u^{-j_{2}^{\prime} r_{2}}\left(u+a_{2} t_{2}^{j_{2}^{\prime} r_{2}}\right)^{e^{\prime}}, z^{p}-z=\left(1+d_{1} t\right) w_{1}^{-g^{\prime}} .
$$

Note that in $\Omega_{u v, K}^{e^{\prime}}$ the function $u$ has no zero or pole and $1+$ $d_{1} t$ has no poles. Thus $\phi_{K}^{\prime}$ has a unique branch point given by the coordinates $u=-a_{2} t^{j_{2}^{\prime} r_{2}}$ and $v=-\left(t^{e^{\prime}-j_{2}^{\prime} r_{2}}\right) / a_{2}=-t^{j_{1}^{\prime} r_{1}} / a_{2}$. This $K$-point specializes to the branch point $(u, v)=(0,0)$ of $\phi_{k}$. Thus $\phi_{R}$ is branched at exactly one $R$-point for this choice of $d_{0}$.

Irreducibility: Consider the equations for the cover $\phi_{R}^{\prime}$ :

$$
w_{1}^{m}=u^{-j_{2}^{\prime} r_{2}}\left(u+a_{2} t^{j_{2}^{\prime} r_{2}}\right)^{e^{\prime}}, z^{p}-z=\left(1+d_{1} t\right) w_{1}^{-g^{\prime}} .
$$

Note that if $m, e^{\prime}$ and $j_{2}^{\prime} r_{2}$ share no common factors then the first equation is irreducible; the second is irreducible since the right-hand side is not of the form $\alpha^{p}-\alpha$. Recall that $j_{1}^{\prime}$ and $j_{2}^{\prime}$ are relatively prime to $m$ and thus to $r_{2}$. Since $\operatorname{gcd}\left(m, j_{2}^{\prime} r_{2}\right)=r_{2}$, the curve $W_{R}$ is irreducible if and only if $1=\operatorname{gcd}\left(e^{\prime}, r_{2}\right)=\operatorname{gcd}\left(r_{1}, r_{2}\right)$. Let $g=$ $\operatorname{gcd}\left(m, j_{1}^{\prime} r_{1}+j_{2}^{\prime} r_{2}\right)=\operatorname{gcd}\left(m, e^{\prime}\right)$. We see that $W_{K}$ is irreducible if and only if $g=1$ from the following equations for the normalization of $\phi_{K}^{\prime}$ : $w_{2}^{g}=1, w_{1}^{m / g}=u^{-j_{2}^{\prime} r_{2} / g}\left(u+a_{2} t_{2}^{j_{2}^{\prime} r_{2}}\right)^{e^{\prime} / g}, z^{p}-z=\left(1+d_{1} t\right) w_{1}^{-g^{\prime}}$.

Ramification information: The first equation indicates that normalization of the generic fibre has $g$ components and thus $g$ points above the branch point each with inertia group $I_{K}=\mathbb{Z} / p \rtimes\left\langle c^{g}\right\rangle$. The second equation indicates that $w_{1}$ is an $\left(e^{\prime} / g\right)$ th power of a uniformizer. Thus the third equation indicates that the lower conductor on the generic fibre is $e^{\prime} g^{\prime} / g=e / g$. Thus the cover of the generic fibre has inertia group $I_{K} \simeq \mathbb{Z} / p \rtimes\left\langle c^{g}\right\rangle=\mathbb{Z} / p \rtimes \mu_{m / g}$ and has conductor $e / g=\left(j_{1} r_{1}+j_{2} r_{2}\right) / g$.

It is more difficult to deform the covers $\phi_{1}$ and $\phi_{2}$ together if $p$ divides $\left(j_{1} r_{1}+j_{2} r_{2}\right) / g$. This is done in the following proposition in the case that $m=1$ and $p \mid\left(j_{1}+j_{2}\right)$. The conductor on the generic fibre will be slightly bigger than $\left(j_{1} r_{1}+j_{2} r_{2}\right) / g$.

Proposition 2.3.5. Let $\phi_{1}: X \rightarrow U, \phi_{2}: Y \rightarrow V$ be $\mathbb{Z} / p$-Galois covers of normal connected germs of curves with conductors $j_{1}$ and $j_{2}$ respectively. Suppose that $p \mid\left(j_{1}+j_{2}\right)$. Let $e=j_{1}+j_{2}+\epsilon$ where $\epsilon=2$ if $p \neq 2$ and $\epsilon=3$ if $p=2$. Then there exists a $\mathbb{Z} / p$-Galois cover $\phi_{R}: W_{R} \rightarrow S^{e} \simeq S_{u v}^{e}$ of irreducible germs of $R$-curves such that:

1. The branch locus of the cover $\phi_{R}$ consists of exactly one $R$-point, denoted $b_{R}$. 
2. After normalization, the pullbacks of the special fibre of $\phi_{R}$ to $U$ and $V$, namely $\iota_{u}^{*} i^{*} \phi_{k}$ and $\iota_{v}^{*} i^{*} \phi_{k}$, are isomorphic to $\phi_{1}$ and $\phi_{2}$ away from the branch point.

3. The generic fibre $\phi_{K}: X_{K} \rightarrow S_{K}^{e}=S^{e} \times_{R} K$ of $\phi_{R}$ is an I-Galois cover of smooth irreducible germs of curves whose branch locus consists of the point $b_{K}=b_{R} \times_{R} K$.

4. The cover $\phi_{K}$ has inertia $\mathbb{Z} / p$ and conductor e over the unique branch point $b_{K}$.

Proof. The proof is essentially the same as for Proposition 2.3.4. For $p \neq 2$ one can deform the equations $x^{p}-x=u^{-j_{1}}$ and $y^{p}-y=v^{-j_{2}}$ using

$$
w=u^{j_{1}+1}+a_{1} v^{j_{2}+1}+d_{0} t, z^{p}-z=\left(u+v+d_{1} t\right) / w .
$$

(Here $a_{1}=a^{m}$.) These equations and the Galois action reduce correctly on the components of the special fibre. In particular,

$$
\bmod (v, t): w_{1} \mapsto u_{1}^{j_{1}+1}, z \mapsto x ; \bmod (u, t): w_{1} \mapsto a v_{1}^{j_{2}+1}, z \mapsto y / a .
$$

Since $u v=t^{e}=t^{j_{1}+j_{2}+2}$, for the same choice of $d_{0}$ as in the proof of Proposition 2.3.4, the equations can be rewritten as:

$$
w=u^{-\left(j_{2}+1\right)}\left(u+t^{j_{2}+1}\right)^{j_{1}+j_{2}+2}, z^{p}-z=\left(u+v+d_{1} t\right) / w .
$$

Since $p \nmid e$, the conductor is equal to $e$.

Notation 2.3.6. Let $G$ be a quasi- $p$ group with Sylow $p$-subgroup $S$. Assume $S \simeq \mathbb{Z} / p$. Let $I \simeq \mathbb{Z} / p \rtimes \mu_{m} \subset G$ and let $r=|G| / m p$. Let $\phi_{1}: X \rightarrow \mathbb{P}_{k}^{1}$ and $\phi_{2}: Y \rightarrow \mathbb{P}_{k}^{1}$ be two (possibly disconnected) $G$-Galois covers each branched at only one point. Let $u$ (respectively $v$ ) be a local parameter at the branch point of $\phi_{1}$ (respectively $\phi_{2}$ ). Suppose the cover $\phi_{i}$ has inertia $I_{i} \simeq \mathbb{Z} / p \rtimes \mu_{m_{i}} \subset I$ and conductor $j_{i}$ for $i=1,2$ above $u=0$ and $v=0$ respectively. Let the genus of $X$ and $Y$ be $g_{1}$ and $g_{2}$ respectively.

Let $P_{R}^{e^{\prime}}$ be an $R$-curve whose generic fibre is isomorphic to $\mathbb{P}_{K}^{1}$, whose special fibre consists of two projective lines $P_{u}$ and $P_{v}$ meeting transversally at a point $b$ where $(u, v)=(0,0)$, and which satisfies $\hat{P}_{R, b} \simeq S_{u v}^{e^{\prime}}$.

The next theorem uses Propositon 2.2.1 and Theorem 1.2.4 to deform the covers $\phi_{1}$ and $\phi_{2}$ to a family of covers $\phi_{R}$ of $P_{R}^{e^{\prime}}$ branched at only one $R$-point. This family can be defined over a variety $\Theta$ of finite type over $k$. We then specialize to a fibre of the family over another $k$-point of $\Theta$ to get a cover $\phi^{\prime}$ with new ramification data.

Theorem 2.3.7. Consider the pair $\left(\phi_{1}, \phi_{2}\right)$ as in Notation 2.3.2 and 2.3.6. Suppose that $\phi_{1}$ and $\phi_{2}$ satisfy the numerical hypotheses. Then there exist $G$-Galois covers of curves $\phi_{R}: Y_{R} \rightarrow P_{R}^{e^{\prime}}$ and $\phi^{\prime}: Y^{\prime} \rightarrow \mathbb{P}_{k}^{1}$ such that:

1. After normalization, the pullbacks of the special fibre of $\phi_{R}$ to $P_{u}$ and $P_{v}$ are isomorphic respectively to $\phi_{1}$ and $\phi_{2}$ away from $b$. 
2. The branch locus of the cover $\phi_{R}$ (respectively $\left.\phi^{\prime}\right)$ consists of exactly one $R$-point denoted $b_{R}$ which specializes to $b$ (respectively of exactly one point $\left.b^{\prime}\right)$.

3. There are $g$ ramification points of $\phi_{K}$ (respectively $\left.\phi^{\prime}\right)$ above the branch point $b_{K}$ (respectively above $\left.b^{\prime}\right)$. These have inertia group $I_{K} \simeq \mathbb{Z} / p \rtimes$ $\left\langle c^{g}\right\rangle=\mathbb{Z} / p \rtimes \mu_{m / g}$ and conductor $e / g=\left(j_{1} r_{1}+j_{2} r_{2}\right) / g$.

4. The curves $Y_{K}$ and $Y^{\prime}$ are smooth of genus $g_{1}+g_{2}-1+|G|-r(p m+$ $\left.g-r_{1}-r_{2}\right) / 2$.

5. Suppose $G_{1}$ and $G_{2}$ are the stabilizers of a connected component of $X$ and $Y$ respectively. Then $Y_{R}$ and $Y^{\prime}$ are connected if $G_{1}$ and $G_{2}$ generate $G$.

Proof. After doing the appropriate set-up, the proof is identical to that of Theorem 2.2.2. Let $X^{*}=P_{R}^{e^{\prime}}$ and let $\mathbb{S}=\{b\}$. Let $S \simeq \mathbb{Z} / p$ be a chosen Sylow $p$-subgroup of $G$. Then there exist points $\eta_{1} \in \phi_{1}^{-1}(u)$ and $\eta_{2} \in \phi_{2}^{-1}(v)$ with inertia groups $I_{i} \simeq S \rtimes \mu_{m_{i}}$. Consider the $I_{i}$-Galois covers of germs of curves $\hat{\phi}_{1}: \hat{X}_{\eta_{1}} \rightarrow U$ and $\hat{\phi}_{2}: \hat{Y}_{\eta_{2}} \rightarrow V$.

Applying Proposition 2.3.4 to the pair $\left(\hat{\phi}_{1}, \hat{\phi}_{2}\right)$ we see there exists an $I$ Galois cover $\hat{\phi}_{R}: W_{R} \rightarrow S^{e^{\prime}}$ with all the desired properties at the unique branch point $b_{K}$. Namely, there are $g$ ramification points above the branch point over $K$. The inertia group at one of these points is of the form $I_{K} \simeq$ $\mathbb{Z} / p \rtimes\left\langle c^{g}\right\rangle$ and has conductor $e / g$. Consider the inclusion $R_{s} \rightarrow A_{s}$ of rings corresponding to the disconnected $G$-Galois cover $\operatorname{Ind}_{I}^{G}\left(\hat{\phi}_{R}\right)$.

Consider the cover $\phi_{k}$ of the special fibre of $P_{R}^{e^{\prime}}$ which restricts to $\phi_{1}$ over $P_{u}$ and to $\phi_{2}$ over $P_{v}$. The covers $\phi_{k}$ and $\operatorname{Ind}_{I}^{G}\left(\hat{\phi}_{R}\right)$ and the isomorphisms given by Proposition 2.3.4 (2) constitute a relative $G$-Galois thickening problem as in Definition 1.2.1. The (unique) solution to this thickening problem (Theorem 1.2.4) yields the $G$-Galois cover $\phi_{R}$. Recall from Definition 1.2.3 that the cover $\phi_{R}$ is isomorphic to $\operatorname{Ind}_{I}^{G}\left(\hat{\phi}_{R}\right)$ over $\hat{P}_{R, b}$. Thus the deformation $\phi_{R}$ has the desired properties near the branch point $b_{R}$. Also, the cover $\phi_{R}$ is isomorphic to the trivial deformation $\phi_{t r}$ of $\phi$ away from $b$, which completes the proof of Properties 1)-3) for $\phi_{R}$. The fact that $Y_{K}$ is smooth follows because both $W_{K}$ and $\phi_{t r}$ are smooth.

If $g^{\prime}$ is the genus of the fibres of $Y_{K}$, the Riemann-Hurwitz formula implies:

$$
\begin{aligned}
& 2 g_{1}-2=-2|G|+r r_{1}\left[\left(p m_{1}-1\right)+j_{1}(p-1)\right], \\
& 2 g_{2}-2=-2|G|+r r_{2}\left[\left(p m_{2}-1\right)+j_{2}(p-1)\right], \\
& 2 g^{\prime}-2=-2|G|+r g[(p m / g-1)+e(p-1) / g] .
\end{aligned}
$$

Using the fact that $e=j_{1} r_{1}+j_{2} r_{2}$ it follows that:

$$
g^{\prime}=g_{1}+g_{2}-1+|G|-r\left(p m+g-r_{1}-r_{2}\right) / 2 .
$$

Note that $p m+g-r_{1}-r_{2}$ is always even. Finally, if $G_{1}$ and $G_{2}$ generate $G$ then the special fibre of $Y_{R}$ is connected. Thus $Y_{K}$ is connected. 
As in the proof of Theorem 2.2.2, the cover $\phi_{R}$ can be defined over $\operatorname{Spec}(\Theta)$ for some $k \neq \Theta \subset R$ of finite type over $k$. Also one can choose a fibre $\phi^{\prime}$ : $Y^{\prime} \rightarrow \mathbb{P}_{k}^{1}$ over a $k$-point of $\Theta$ so that $\phi^{\prime}$ is a $G$-Galois cover and $Y^{\prime}$ is smooth with the same number of connected components as $Y_{K},[\mathbf{2}$, Proposition 9.29]. Properties (2)-(5) for $\phi^{\prime}$ follow immediately from the corresponding properties for $\phi_{K}$.

Remark 2.3.8. These patching results do not need to be restricted to the case $X=\mathbb{P}_{k}^{1}$ or the case of only one branch point. In general, one can consider $G$-Galois covers $\phi_{1}: Y_{1} \rightarrow X_{1}$ and $\phi_{2}: Y_{2} \rightarrow X_{2}$. Suppose $\phi_{1}$ and $\phi_{2}$ each have a branch point with inertia group contained in $I \simeq \mathbb{Z} / p \rtimes \mu_{m}$ whose ramification data satisfies the numerical hypotheses. Using Proposition 2.3.4, one can construct a cover $\phi: Z \rightarrow W$ with specified inertia behavior above one point. The genus of $W$ will be the sum of genus $\left(X_{1}\right)$ and genus $\left(X_{2}\right)$ and $\phi$ will be branched at $\left|B_{1}\right|+\left|B_{2}\right|-1$ points.

Remark 2.3.9. One would like to know whether the above constructions are optimal in the following sense: Given the covers $\phi_{1}$ and $\phi_{2}$, with upper jumps $\sigma_{1}=j_{1} / m_{1}$ and $\sigma_{2}=j_{2} / m_{2}$, in Theorem 2.3.7 we construct a deformation so that the generic fibre is a cover $\phi_{K}$ branched at exactly one point with upper jump $\sigma_{\eta}=\sigma_{1}+\sigma_{2}$; would it have been possible to get any smaller upper jump on the generic fibre? The key formula [7, Theorem 3.11] indicates that the result in Theorem 2.3.7 is almost optimal since the upper jump on the generic fibre must satisfy $\sigma_{\eta} \geq \sigma_{1}+\sigma_{2}-1$. In other words, the singularity for $\phi_{k}$ is not too severe. The following lemma gives another way to measure this singularity.

Consider the cover $\phi_{R}: Y_{R} \rightarrow \mathbb{P}_{R}^{1}$ constructed in Theorem 2.2.2 (respectively $\phi_{R}: Y_{R} \rightarrow P_{R}^{e^{\prime}}$ constructed in Theorem 2.3.7 or Corollary 2.3.1). Choose $y \in \phi_{R}^{-1}\left(\infty_{k}\right)$ (respectively $\left.\phi_{R}^{-1}(0,0)\right)$. Let $\pi_{y}: \widetilde{Y}_{y, k} \rightarrow \hat{Y}_{y, k}$ be the normalization of $\hat{Y}_{y, k}$ and let $\hat{\mathcal{O}}_{y} \rightarrow \widetilde{\mathcal{O}}_{y}$ be the corresponding extension of rings. Let $\delta_{y}=\operatorname{dim}_{k}\left(\widetilde{\mathcal{O}}_{y} / \mathcal{O}_{y}\right)$ and let $m_{y}=\# \pi_{Y}^{-1}(y)$. Let $\mu_{y}=2 \delta_{y}-m_{y}+1$.

\section{Lemma 2.3.10.}

i) In Theorem 2.2.2, $\mu_{y}=\left(j_{\eta}-j_{b}\right)(p-1)=i m(p-1)$.

ii) In Theorem 2.3.7 and Corollary 2.3.1 (2-3), $\mu_{y}=1+m p-g$.

iii) In Corollary 2.3.1 (1), $\mu_{y}=p+\epsilon(p-1)$.

Proof. The proof uses a formula of Kato [6] which compares the local ramification and the singularities for the cover $\hat{\phi}_{R}$ of germs of curves. The details are omitted.

\section{Applications to ramification questions.}

Let $G$ be a finite quasi- $p$ group. Let $S$ be a chosen Sylow $p$-subgroup of $G$ and suppose $S$ has order $p$. Let $k$ be an algebraically closed field of characteristic 
$p$. All covers in this section are smooth, connected and proper. We now show that for all sufficiently large $j \in \mathbb{N}$ with $p \nmid j$, there exists a $G$-Galois cover $\phi: Y \rightarrow \mathbb{P}_{k}^{1}$ branched at only one point with inertia $\mathbb{Z} / p$ and conductor $j$. The method is to first show the existence of such a cover with small conductor under an additional hypothesis on $G$. We then use group theory to determine which conductors are sufficiently large enough to realize with formal patching.

3.1. $\boldsymbol{P}$-Weight. In this section, we measure the complexity of the group G.

Definition 3.1.1. Let $G(S) \subset G$ be the subgroup generated by all proper quasi- $p$ subgroups $G^{\prime}$ such that $G^{\prime} \cap S$ is a Sylow $p$-subgroup of $G^{\prime}$. The group $G$ is $p$-pure if $G(S) \neq G$.

Note that this definition is independent of the choice of $S$. This condition was introduced in [10]. Note that when $|S|=p$, then $G$ is $p$-pure if and only if $G$ is not generated by all proper quasi- $p$ subgroups $G^{\prime} \subset G$ such that $S \subset G^{\prime}$. Some examples of $p$-pure quasi- $p$ groups with $|S|=p$ are $\operatorname{PSL}_{2}\left(\mathbb{F}_{p}\right)$, and the semi-direct product $(\mathbb{Z} / r \mathbb{Z})^{l} \rtimes \mathbb{Z} / p$ where the action is irreducible. When $p=11, M_{11}$ and $M_{22}$ are quasi-11 and 11-pure. Every finite quasi- $p$ group can be generated from $p$-pure ones.

Definition 3.1.2. Consider all subgroups $G^{\prime} \subset G$ such that $G^{\prime}$ is quasi-p and $p$-pure and such that $G^{\prime} \cap S$ is a Sylow $p$-subgroup of $G^{\prime}$. The $p$-weight $\omega(G)$ of $G$ is the minimal number of such subgroups $G^{\prime}$ of $G$ which are needed to generate $G$.

Lemma 3.1.3. The p-weight $\omega(G)$ of $G$ is a finite number independent of the choice of $S$.

Proof. The proof uses induction on $|G|$ to show that $G$ can be generated by $p$-pure quasi- $p$ subgroups $G^{\prime \prime}$ with $G^{\prime \prime} \cap S=\operatorname{Syl}_{p}\left(G^{\prime \prime}\right)$. This statement is true if $G \simeq \mathbb{Z} / p$. For then $G$ contains no proper quasi- $p$ subgroups and so $\{1\}=G(S) \neq G$. Thus $G \simeq \mathbb{Z} / p$ is $p$-pure and $\omega(G)=1$.

Now given $G$, suppose that the hypothesis is true for any quasi- $p$ group $G^{\prime}$ such that $p \leq\left|G^{\prime}\right|<|G|$. If $G(S) \neq G$ then $G$ is $p$-pure and so $\omega(G)=1$.

If $G(S)=G$ then by definition $G$ is generated by its proper quasi- $p$ subgroups $G^{\prime} \subset G$ with $G^{\prime} \cap S=\operatorname{Syl}_{p}\left(G^{\prime}\right)$. Since $\left|G^{\prime}\right|<|G|$ the induction hypothesis states that each $G^{\prime}$ is generated by $p$-pure quasi- $p$ subgroups $G^{\prime \prime}$ with $G^{\prime \prime} \cap \operatorname{Syl}_{p}\left(G^{\prime}\right)=\operatorname{Syl}_{p}\left(G^{\prime \prime}\right)$. Note that $\operatorname{Syl}_{p}\left(G^{\prime \prime}\right)=G^{\prime \prime} \cap\left(G^{\prime} \cap S\right)=G^{\prime \prime} \cap S$. Thus each $G^{\prime \prime}$ satisfies the necessary conditions and the collection of $G^{\prime \prime}$ generate $G$. Thus the $p$-weight is the minimum size among all sets of $p$-pure quasi- $p$ subgroups $G^{\prime \prime}$ with $S \cap G^{\prime \prime}=\operatorname{Syl}_{p}\left(G^{\prime \prime}\right)$ which generate $G$.

To show that $\omega(G)$ is independent of $S$, consider another Sylow $p$-subgroup $S_{0}$ of $G$. Let $\omega_{0}(G)$ be the $p$-weight with respect to $S_{0}$. Since the Sylow 
$p$-subgroups are all conjugate, there exists some $g \in G$ with $S_{0}=g S g^{-1}$. Suppose $G$ is generated by a set $\left\{G^{\prime \prime}\right\}$ of $p$-pure quasi- $p$ subgroups with $S \cap G^{\prime \prime}=\operatorname{Syl}_{p}\left(G^{\prime \prime}\right)$. Note that $S_{0} \cap\left(g G^{\prime \prime} g^{-1}\right)=\operatorname{Syl}_{p}\left(g G^{\prime \prime} g^{-1}\right)$. Each subgroup $g G^{\prime \prime} g^{-1}$ is still quasi- $p$ and $p$-pure (with respect to its Sylow). Also $G$ is generated by the set of $g G^{\prime \prime} g^{-1}$. Thus $\omega_{0}(G) \leq \omega(G)$. Reversing the roles of $S$ and $S_{0}, \omega(G) \leq \omega_{0}(G)$.

3.2. Conductors. Let $\phi: Y \rightarrow \mathbb{P}_{k}^{1}$ be a $G$-Galois cover which is branched at only one point. Such a cover exists if and only if $G$ is a quasi- $p$ group which means that $G$ is generated by $p$-groups, $[\mathbf{1 0}]$. Suppose $\phi$ has inertia group $I \simeq \mathbb{Z} / p \rtimes \mu_{m}$ and conductor $j$. When $G \neq \mathbb{Z} / p$, there is a small set of values $j_{\min }(I)$, depending only on $I$, consisting of the minimal possible conductors for $\phi$. Let $n^{\prime}$ be the order of the prime-to- $p$ part of the center of $I$. Let $n$ be such that $m=n n^{\prime}$.

Definition 3.2.1. Define $j_{\min }(I)=\left\{j_{\min }(I, a) \mid 1 \leq a \leq n, \operatorname{gcd}(a, n)=1\right\}$ where $j_{\min }(I, a)=2 m+n^{\prime}$ if $a=1$ and $n=p-1$ and $j_{\min }(I, a)=m+a n^{\prime}$ otherwise.

A geometric interpretation for the set $j_{\min }(I)$ is that $\phi$ has a non-isotrivial deformation in equal characteristic $p$ if and only if $j \notin j_{\min }(I)$, [8, Theorem 3.1.11]. Suppose $1 \leq a \leq n$ and $j \equiv a n^{\prime} \bmod m$. If $G \neq \mathbb{Z} / p$ then $j \geq$ $j_{\min }(I, a)$, by [8, Lemma 1.4.3]. Note that if $j \in j_{\min }(I)$ then $p \nmid j$ and $j \leq m(2+1 /(p-1))$.

Theorem 3.2.2. Let $G$ be a finite p-pure quasi-p group whose Sylow psubgroups have order $p \neq 2$. For some $I \simeq \mathbb{Z} / p \rtimes \mu_{m} \subset G$ and some $j \in j_{\min }(I)$, there exists a $G$-Galois cover $\phi: Y \rightarrow \mathbb{P}_{k}^{1}$ of smooth connected curves branched at only one point over which it has inertia group $I$ and conductor $j$. In particular, genus $(Y) \leq 1+|G|(p-1) / 2 p$.

Proof. For the convenience of the reader we briefly recall the outline of the proof from [7, Theorem 4.5]. By Abhyankar's Conjecture [10, 6.5.3], for some $I_{0}$ of the form $\mathbb{Z} / p \rtimes \mu_{m_{0}}$ and some $j_{0}$, there exists a $G$-Galois cover $\phi_{0}: Y_{0} \rightarrow \mathbb{P}_{k}^{1}$ with group $G$ which is branched at only one point with inertia group $I_{0}$ and conductor $j_{0}$. If $j_{0} \notin j_{\min }(I)$, there exists a non-isotrivial deformation of $\phi_{0}$ in equal characteristic $p$ by [8, Theorem 3.1.11]. This deformation yields a cover $\phi_{K}$ with bad reduction by [8, Theorem 3.3.7].

Let $\phi: Y \rightarrow X$ be the stable model of $\phi_{K}$. See [7, Section 3] for information on the structure of $\phi$, which is very similar to that in the unequal characteristic case in [10, Section 6], [11, Sections 2-3], and [12]. In particular, the special fibre $X_{k}$ is a tree of projective lines and the restriction of $\phi$ over any terminal component of $X_{k}$ is separable. Since $G$ is $p$-pure and has no (nontrivial) normal $p$-subgroups, for some terminal component $P_{b}$ of $X_{k}$, the curve $Y_{b}=\phi^{-1}\left(P_{b}\right)$ is connected. For this component, the restriction $\phi_{b}: Y_{b} \rightarrow P_{b} \simeq \mathbb{P}_{k}^{1}$ is a $G$-Galois cover branched at only one 
point. If $\phi_{b}$ has inertia group $I_{b} \simeq \mathbb{Z} / p \rtimes \mu_{m_{b}} \subset N_{G}(S)$ and conductor $j_{b}$ then $j_{b} / m_{b}<j_{0} / m_{0}$ by [7, Theorem 3.11]. We reiterate this process until finding such a cover with inertia group $I_{b}=\mathbb{Z} / p \rtimes \mu_{m_{b}}$ and conductor $j_{b}$ satisfying $j_{b} / m_{b} \leq 2+1 /(p-1)$ which implies $j_{b} \in j_{\min }(I)$. The condition on $\operatorname{genus}(Y)$ follows directly from Definition 3.2.1 and the Riemann-Hurwitz formula.

Lemma 3.2.3. Suppose there exists a G-Galois cover $\phi: Y \rightarrow \mathbb{P}_{k}^{1}$ branched at only one point with inertia group $I \simeq \mathbb{Z} / p \rtimes \mathbb{Z} / m$ and conductor $j$. Then for any $I^{\prime} \subset I$ of the form $I^{\prime}=\mathbb{Z} / p \rtimes \mu_{m^{\prime}}$ and for any $j^{\prime} \equiv j \bmod m^{\prime}$ with $j^{\prime} \geq j$ and $p \nmid j^{\prime}$, there exists a G-Galois cover $\phi^{\prime}: Y \rightarrow \mathbb{P}_{k}^{1}$ of smooth connected curves branched at only one point with inertia $I^{\prime}$ and conductor $j^{\prime}$.

Proof. Let $r$ be the index of $I^{\prime}$ in $I$. Consider the cover $f: X \rightarrow \mathbb{P}_{k}^{1}$ which is cyclic of order $r$ and branched at 0 and $\infty$. By Abhyankar's Lemma, the cover $f^{*} \phi$ is a $G$-Galois cover $\phi^{\prime}: Y^{\prime} \rightarrow \mathbb{P}_{k}^{1}$ which is branched at only one point with inertia group $\mathbb{Z} / p \rtimes \mu_{m^{\prime}}$. The cover $f^{*} \phi$ is connected since $f$ and $\phi$ are disjoint. The conductor of $f^{*} \phi$ still equals $j$. Thus the statement is immediate from Theorem 2.2.2.

Let $G$ be a quasi- $p$ group with $|S|=p$ and with $p$-weight $\omega$. By Lemma 3.1.3, $G$ can be generated by a collection of $\omega$ proper $p$-pure quasi- $p$ subgroups $G^{\prime}$ such that $G^{\prime} \cap S=\operatorname{Syl}_{p}\left(G^{\prime}\right)$. We give sufficient conditions for the conductor of a $G$-Galois cover $\phi: Y \rightarrow \mathbb{P}_{k}^{1}$ branched at only one point with inertia $\mathbb{Z} / p$.

Theorem 3.2.4. Let $G$ be a finite quasi-p group whose Sylow p-subgroups have order $p \neq 2$. Let $\omega$ be the p-weight of $G$. Let $m_{e}$ be the exponent of the normalizer $N_{G}(S)$ of $S$ in $G$ divided by $p$. Let $j \in \mathbb{N}^{+}$satisfy $\operatorname{gcd}(j, p)=1$. Suppose $j \geq m_{e}(2+1 /(p-1)) \omega$ if $p \nmid \omega$ and $j \geq m_{e}(2+1 /(p-1)) \omega+2$ if $p \mid \omega$. Then there exists a $G$-Galois cover $\phi: Y \rightarrow \mathbb{P}_{k}^{1}$ of smooth connected curves which is branched at only one point over which it has inertia $\mathbb{Z} / p$ and conductor $j$.

Proof. Note that $m_{e}(2+1 /(p-1))$ is not necessarily an integer. In this proof the phrase "cover of this type" indicates that the cover in question is a smooth connected cover of the projective line branched at only one point with inertia $I=\mathbb{Z} / p$.

By Theorem 2.2.2, given $G$ as above it is sufficient to prove the following: For some $J \in \mathbb{Z}$ such that $p \nmid J$ and $J \leq m_{e}(2+1 /(p-1))$ there exists a $G$-Galois cover $\phi$ of this type with conductor $j=J \omega$ if $p \nmid \omega$ and conductor $j=J \omega+2$ if $p \mid \omega$. The proof will proceed by induction on $\omega$.

If $\omega=1$ then $G$ is quasi- $p$ and $p$-pure. By Theorem 3.2.2, for some $I \simeq \mathbb{Z} / p \rtimes \mu_{m} \subset G$ there exists a $G$-Galois cover $\phi: Y \rightarrow \mathbb{P}_{k}^{1}$ branched at only one point with inertia group $I$ and conductor $j \in j_{\min }(I)$. Recall 
that if $j \in j_{\min }(I)$ then $j=m(2+1 /(p-1))$ or $j \leq 2 m$. Also $m \leq m_{e}$. Let $I^{\prime}=\mathbb{Z} / p$. By Lemma 3.2.3, there exists a $G$-Galois cover $\phi^{\prime}: Y \rightarrow \mathbb{P}_{k}^{1}$ branched at only one point with inertia $\mathbb{Z} / p$ and conductor $j$. Choose $J=j$ and note that $p \nmid J$ and $J \leq m_{e}(2+1 /(p-1))$.

Now suppose that $\omega>1$. By the inductive hypothesis, for all quasi- $p$ groups $G^{\prime}$ having $p$-weight $\omega^{\prime}$ where $\omega^{\prime}<\omega$ and $p \nmid \omega^{\prime}$, there exists $J^{\prime}$ such that $\operatorname{gcd}\left(J^{\prime}, p\right)=1$ and such that $J^{\prime} \leq m_{e^{\prime}}\left(2+1 /(p-1)\right.$ ) (where $m_{e^{\prime}}$ is the exponent of $N_{G^{\prime}}(S)$ divided by $p$ ) and there exists a $G^{\prime}$-Galois cover $\phi^{\prime}$ of this type with conductor $j^{\prime}=J^{\prime} \omega^{\prime}$.

Choose $w_{1} \geq 1$ and $w_{2} \geq 1$ such that $p \nmid w_{1} w_{2}$ and $w_{1}+w_{2}=\omega$. (If $\omega \not \equiv 1 \bmod p$, then choose $w_{1}=1$ and $w_{2}=\omega-1$. If $\omega \equiv 1 \bmod p$, then choose $w_{1}=2$ and $w_{2}=\omega-2$.)

Since $G$ has $p$-weight $\omega>1, G$ can be generated by $\omega$ proper $p$-pure quasi- $p$ groups $G_{1}^{\prime}, \ldots, G_{\omega}^{\prime}$ with $S \subset G_{i}^{\prime}$ for all $1 \leq i \leq \omega$. Let $G_{1} \subset G$ be the subgroup generated by $G_{i}^{\prime}$ for $1 \leq i \leq w_{1}$. Let $G_{2} \subset G$ be the subgroup generated by $G_{i}^{\prime}$ for $w_{1}+1 \leq i \leq w_{2}$. Then $G_{1}$ and $G_{2}$ are quasi- $p$ groups since they are generated by quasi- $p$ groups. For $i=1,2$, the order of a Sylow $p$-subgroup of $G_{i}$ is $p$ since $S \subset G_{i} \subset G$. By construction, $\omega\left(G_{1}\right) \leq w_{1}$ and $\omega\left(G_{2}\right) \leq w_{2}$. But in fact, $\omega\left(G_{1}\right)=w_{1}$ and $\omega\left(G_{2}\right)=w_{2}$ since the $p$-weight of $G$ is only $\omega$. For $i=1,2$, let $m_{e_{i}}$ be the exponent of $N_{G_{i}}(S)$ divided by $p$.

By the inductive hypothesis, for $i=1,2$, there exists $J_{i}$ such that $p \nmid J_{i}$ and $J_{i} \leq m_{e_{i}}(2+1 /(p-1))$ and there exists a $G_{i}$-Galois cover $\phi_{i}$ of this type with conductor $J_{i} w_{i}$. Let $m_{e}$ be the exponent of the normalizer of $N_{G}(S)$ divided by $p$ and note that $m_{e} \geq m_{e_{i}}$. Let $J=\max \left\{J_{1}, J_{2}\right\}$ and note that $p \nmid J$ and $J \leq m_{e}(2+1 /(p-1))$. Using Theorem 2.2.2 to increase the conductor of $\phi_{i}$ for $i=1,2$ we find a $G_{i}$-Galois cover $\phi_{i}^{\prime}$ of this type with conductor $J w_{i}$.

By Corollary 2.3.1 (1), in the case that $p \nmid \omega$, there exists a $G$-Galois cover $\phi$ of this type with conductor $j=J w_{1}+J w_{2}=J \omega$; in the case that $p \mid \omega$, there exists a $G$-Galois cover $\phi$ of this type with conductor $j=$ $J w_{1}+J w_{2}+2=J \omega+2$. (In particular, the covers are connected since $G_{1}$ and $G_{2}$ generate $G$, Theorem 2.3 .7 (5).) This completes the proof by induction.

Example 3.2.5. Let $G=\mathrm{PSL}_{2}\left(\mathbb{F}_{p}\right)$. Then $G$ is quasi- $p$ and $p$-pure and its Sylow $p$-subgroups have order $p$. The normalizer of a Sylow is of the form $I^{*} \simeq \mathbb{Z} / p \rtimes \mu_{m^{*}}$ where $m^{*}=(p-1) / 2$ and $\mu_{m^{*}}$ acts faithfully on $\mathbb{Z} / p$. By Corollary 3.2.2, for some $m \mid m^{*}$, there exists a $G$-Galois cover $\phi: Y \rightarrow \mathbb{P}_{k}^{1}$ of smooth connected curves branched only at $\infty$ with inertia $I \simeq \mathbb{Z} / p \rtimes \mu_{m}$ and conductor $j \in j_{\min }(I)$. In this case $j \leq 2 m \leq 2 m^{*}<p$.

Acknowledgements. I would like to thank D. Harbater and K. Stevenson for their help improving earlier drafts of this work. 


\section{References}

[1] I. Bouw and R. Pries, Rigidity, reduction, and ramification, to appear in Math. Ann.

[2] M. Fried and M. Jarden, Field Arithmetic, Springer-Verlag, Berlin, 1986, MR 89b:12010, Zbl 0625.12001.

[3] D. Harbater, Formal patching and adding branch points, Amer. J. Math., 115(3) (1993), 487-508, MR 94f:14024, Zbl 0790.14027.

[4] _ Abhyankar's conjecture on Galois groups over curves, Invent. Math., 117(1) (1994), 1-25, MR 95i:14029, Zbl 0805.14014.

[5] D. Harbater and K. Stevenson, Patching and thickening problems, J. Algebra, 212(1) (1999), 272-304, MR 2000b:14004, Zbl 0944.14013.

[6] K. Kato, Vanishing cycles, ramification of valuations, and class field theory, Duke Math. J., 55(3) (1987), 629-659, MR 88k:11083, Zbl 0665.14005.

[7] R. Pries, Conductors of wildly ramified covers, I, C.R. Acad. Sci. Paris Sér. I Math., 335(1) (2002), 481-484, CMP 1937117.

[8] _ Families of wildly ramified covers of curves, Amer. J. Math., 124(4) (2002), 737-768, CMP 1914457.

[9] _ Construction of covers with formal and rigid geometry, in 'Courbes semistables et groupe fondamental en géométrie algébrique (Luminy, 1998),' 157-167, Birkhäuser, Basel, 2000, MR 2001e:14026, Zbl 0978.14018.

[10] M. Raynaud, Revêtements de la droite affine en caractéristique $p>0$ et conjecture d'Abhyankar, Invent. Math., 116(1-3) (1994), 425-462, MR 94m:14034, Zbl 0798.14013.

[11] Spécialisation des revêtements en caractéristique $p>0$, Ann. Sci. École Norm. Sup. (4), 32(1) (1999), 87-126, MR 2000e:14016, Zbl 0999.14004.

[12] M. Saïdi, Raynaud's proof of Abhyankar's conjecture for the affine line, in 'Courbes semi-stables et groupe fondamental en géométrie algébrique,' Progress in Mathematics, 187, 249-265, Birkhäuser, 2000.

[13] J.-P. Serre, Corps Locaux, Hermann, 1968, MR 50 \#7096, Zbl 0174.24301.

Received June 13, 2002 and revised November 2, 2002.

Department of Mathematics

Columbia University

NEW YORK, NY 10027

E-mail address: pries@math.columbia.edu 